\title{
Transcription-controlled gene therapy against tumor angiogenesis
}

\author{
Shoshana Greenberger, ${ }^{1,2,3}$ Aviv Shaish, 1,2,3 Nira Varda-Bloom, 1,2 Keren Levanon,1,2 Eyal Breitbart, ${ }^{3}$ \\ Iris Goldberg, ${ }^{2,4}$ Iris Barshack, ${ }^{2,4}$ Israel Hodish, ${ }^{1,2}$ Niva Yaacov, ${ }^{3}$ Livnat Bangio, ${ }^{3}$ Tanya Goncharov, ${ }^{5}$ \\ David Wallach, ${ }^{5}$ and Dror Harats ${ }^{1,2,3}$
${ }^{1}$ Institute of Lipid and Atherosclerosis Research, Sheba Medical Center, Tel Hashomer, Israel. ${ }^{2}$ Sackler School of Medicine, Tel Aviv University, Tel Aviv, Israel. ${ }^{3}$ Vascular Biogenics Ltd., Or Yehuda, Israel. ${ }^{4}$ Institute of Pathology, Sheba Medical Center, Tel Hashomer, Israel. ${ }^{5}$ Department of Membrane Research and Biophysics, Weizmann Institute of Science, Rehovot, Israel.

\begin{abstract}
A major drawback of current approaches to antiangiogenic gene therapy is the lack of tissue-specific targeting. The aim of this work was to trigger endothelial cell-specific apoptosis, using adenoviral vector-mediated delivery of a chimeric death receptor derived from the modified endothelium-specific pre-proendothelin-1 (PPE-1) promoter. In the present study, we constructed an adenovirus-based vector that targets tumor angiogenesis. Transcriptional control was achieved by use of a modified endothelium-specific promoter. Expression of a chimeric death receptor, composed of Fas and TNF receptor 1, resulted in specific apoptosis of endothelial cells in vitro and sensitization of cells to the proapoptotic effect of TNF- $\alpha$. The antitumoral activity of the vectors was assayed in two mouse models. In the model of B16 melanoma, a single systemic injection of virus to the tail vein caused growth retardation of tumor and reduction of tumor mass with central tumor necrosis. When the Lewis lung carcinoma lung-metastasis model was applied, i.v. injection of vector resulted in reduction of lungmetastasis mass, via an antiangiogenic mechanism. Moreover, by application of the PPE-1-based transcriptional control, a humoral immune response against the transgene was avoided. Collectively, these data provide evidence that transcriptionally controlled, angiogenesis-targeted gene therapy is feasible.
\end{abstract}

\section{Introduction}

Angiogenesis, the formation of new capillaries by budding from existing vessels, occurs in tumors and permits their growth, invasiveness, and the spread of metastasis (1). The antiangiogenic approach to antitumor treatment targets these new vessels because of their accessibility by i.v. administration, the paucity of mutations, and the amplification effect on tumor killing (2). The endothelial cells (ECs) of the newly formed blood vessels are affected by antiangiogenic factors, such as angiostatin and endostatin, that trigger their apoptosis (3). In contrast, proangiogenic factors like bFGF and VEGF contribute to cell survival (4). The induction of direct and specific EC apoptosis is assumed to disrupt the balance between the anti- and proapoptotic signals and to thereby cut off the tumor's blood supply.

Transductional targeting of ECs by gene therapy approaches was hampered by the inefficiency of the vascular-specific promoters used $(5,6)$. In the present work, we used a modified pre-proendothelin-1 (PPE-1) promoter to direct gene expression to ECs. PPE1 is the precursor protein for endothelin-1, a 21-amino acid peptide that acts as a potent vasoconstrictor and smooth muscle cell mitogen and is synthesized by ECs (7). The murine PPE- 1 promoter contains a hypoxia-responsive element that increases its expression under hypoxic conditions (8). An augmented transcriptional rate is noted also in the presence of cytokines like TNF- $\alpha$ (9) and

Nonstandard abbreviations used: adenovirus 5 (Ad5); bovine aortic endothelial cell (BAEC); cytomegalovirus (CMV); endothelial cell (EC); Fas-associated death domain (FADD); Fas-chimera (Fas-c); human umbilical vein EC (HUVEC); Lewis lung carcinoma (LLC); normal skin fibroblast (NSF); pre-proendothelin (PPE); receptorinteracting protein (RIP); TNF receptor 1 (TNFR1).

Conflict of interest: S. Greenberger, A. Shaish, E. Breitbart, N. Yaacov, L. Bangio, and D. Harats are employees of Vascular Biogenics Ltd., a biotechnology company developing anti-cancer gene therapy products.

Citation for this article: J. Clin. Invest. 113:1017-1024 (2004).

doi:10.1172/JCI200420007.
TGF- $\beta$ (10). Higher expression levels of genes directed by the PPE1 promoter are therefore expected in the hypoxic and cytokine-rich microenvironment of tumor angiogenesis. In previous work, we demonstrated EC-specific expression of transgenes using the murine PPE-1 in transgenic mice (11). An adenovirus-based vector containing the murine PPE-1 promoter induced high and specific expression in ECs both in vitro and in vivo in tumor angiogenesis (12). We used a modified PPE-1 promoter, constructed in our laboratory and termed PPE-1-3x, which contains three copies of the EC-positive regulatory elements (13). PPE-1-3x was demonstrated to express genes efficiently and specifically in angiogenic vessels $(\mathrm{N}$. Varda-Bloom, manuscript submitted for publication).

Another major obstacle on the road to efficient adenovirus-mediated gene therapy is the transient expression observed in various in vivo models. Limited transgene expression is attributed both to gene silencing (14) and to an immunological attack on the vector-containing cells (15). Although neutralizing antibodies develop against both the viral antigens and the transgene, it was demonstrated that the response directed against the foreign transgene-encoded proteins is the major determinant of the stability of transgene expression (16). A recent report showed that the immune response against the transgene can be avoided by use of a liver-specific promoter (17). Since ECs perform poorly as APCs $(18,19)$, we examined gene expression under the control of the PPE-1-3x promoter to see if it would reduce the humoral response against the transgene and increase transgene stability.

A prerequisite for effective antivascular gene therapy is the use of a potent "killer" gene. In this regard, a death receptor like Fas is an attractive candidate. Fas (CD95) and TNF receptor 1 (TNFR1; p55) are transmembrane proteins, members of the TNF receptor superfamily (20). Fas-FasL interaction was shown to play a crucial role in the control of angiogenesis (21). In addition, recent studies demonstrated that Fas is upregulated during remodeling of vas- 
cular endothelium and is required for the inhibitory action of antiangiogenic factors (22). Gene transfer of Fas and FasL induces apoptosis in several tumor models in vivo (23). However, injections of recombinant FasL or anti-Fas mAb's to animals also rapidly induced massive degeneration of hepatocytes, necrosis, and hemorrhages and ultimately led to death (24). Boldin et al. developed a chimeric receptor constructed of the extracellular portion of TNFR1 and the transmembrane and intracellular region of Fas (25). This receptor, termed Fas-chimera (Fas-c), can initiate a Fasinduced apoptotic pathway by binding not to FasL, but to TNF- $\alpha$, which is abundant in the tumor's microenvironment (26).

Putting all this information together, we assumed that the use of an angiogenesis-specific promoter in combination with a transgene that is specifically activated in the tumor's microenvironment would provide us with two levels of tumor specificity. The aim of the present work was to generate a replication-deficient adenovirus vector, expressing TNF- $\alpha$-triggered Fas under the control of PPE$1-3 x$, as an antitumor angiogenesis tool.

\section{Methods}

Cell culture. The following cell lines were used: Bovine aortic ECs (BAECs) were a kind gift from N. Savion (Goldschleger Eye Institute, Sheba Medical Center). Human embryonic kidney cells (293 cells) were purchased from American Type Culture Collection (Rockville, Maryland, USA). Passages 20-26 of 293 cells were used. D122-96 (Lewis lung carcinoma [LLC]) cells were kindly provided by L. Eizenbach (Weizmann Institute of Science). HeLa cells and B16 melanoma cells were a kind gift from Y. Keisary (Tel Aviv University). Normal skin fibroblasts (NSFs) were cultured from the arm skin of healthy donors. Human umbilical vein ECs (HUVECs) were produced as previously described (27). Cells were cultured in DMEM supplemented with $10 \%$ FCS and $100 \mathrm{U} / \mathrm{ml}$ penicillin/ streptomycin. HUVECs were grown with RPMI 1640 and 10\% FCS (Biological Industries Ltd., Beit Haemek, Israel). Fifty nanograms per milliliter of bFGF was added to BAECs. All the cells were maintained at $37^{\circ} \mathrm{C}$ in a $5 \% \mathrm{CO}_{2}$-humidified incubator.

Expression constructs. Mammalian expression constructs were made in pcDNA3 (Invitrogen Corp., Carlsbad, California, USA). Human cDNA of TNFR1 (p55), MORT1, receptor-interacting protein (RIP), CASH, caspase-3, caspase-9, and Fas-c were subcloned into the multiple cloning site of pcDNA3. Fas-c is a fusion of two death receptors constructed from the extracellular region of TNFR1 and the transmembrane and intracellular regions of Fas (25).

Transfection experiments. BAECs and 293 cells were cultured in 24well plates on cover glasses to $60-70 \%$ confluence. Cotransfection was done using $0.4 \mu \mathrm{g} /$ well of expression construct and $0.04 \mu \mathrm{g} /$ well of pEGFP-C1 vector (CLONTECH Laboratories Inc., Palo Alto, California, USA). Lipofectamine and Lipofectamine Plus in OptiMEM (Invitrogen Life Technologies, Carlsbad, California, USA) were used for 293 cells and for BAECs, respectively. After 4 hours of incubation at $37^{\circ} \mathrm{C}$, the transfection mixture was replaced with growth medium. At 24 hours after transfection, cover glasses were placed on slides, and GFP was detected by fluorescent microscopy. The apoptotic cells were identified based on the typical morphology: cells were small and round compared with normal cells. The percentage of apoptotic cells out of the total GFP-expressing cells was calculated. Electron microscopy of cells transfected with proapoptotic genes was used as a means of evaluating apoptotic activity, as previously described (28).

Construction of adenoviral vectors. Ad-PPE-Fas-c, Ad-CMV-Fas-c, and Ad-PPE-luciferase (Ad-PPE-luc) vectors were generated by homologous recombination as previously described (29). Ad-PPE-Fas-c contains a modified PPE-1 promoter developed in our laboratory, termed PPE$1-3 x$ (30). The presence of the PPE-Fas-c insert was verified by PCR using the oligonucleotide pairs $5^{\prime}$-CTCTTGATTCTTGAACTCTG-3' (upstream, PPE-1 primer) and 5'-ACAAGTAGGTTCCTTTGTG-3' (downstream, Fas-c primer), and 5'-AGATCTCTTCTTGCACAGTG$3^{\prime}$ (upstream, TNFR1 primer) and 5'-TGAAGTTGATGCCAATTACG$3^{\prime}$ (downstream, Fas primer).

RT-PCR assays. BAECs were cultured in $60-\mathrm{mm}$ dishes to about $70 \%$ confluence. They were transduced with Ad-PPE-Fas-c. Control cells were not transduced. Cells were harvested for total RNA purification 2 days after transduction. All the following procedures were carried out with sterile, RNase-free solutions, reagents, and plasticware. Total RNA was isolated using the RNeasy Mini Kit (QIAGEN GmbH, Hilden, Germany) and quantitated by spectrophotometric analysis at wavelengths of 260 and $280 \mathrm{~nm}$. To assure the quality of the RNA isolates, several samples were analyzed by electrophoresis in denaturing agarose gel. A volume containing $1 \mathrm{ng}$ of total RNA of each sample was used for RT-PCR using reagents from the Titan One Tube RT-PCR kit (Roche Diagnostics GmbH, Mannheim, Germany), together with control RNA supplied by the kit. The reaction mixture was prepared according to the kit protocol and contained the primers 5'-AGATCTCTTCTTGCACAGTG$3^{\prime}$ (upstream, from the TNFR1 component of the chimeric transgene) and 5'-TGAAGTTGATGCCAATTACG-3' (downstream, from the Fas component of the chimeric transgene). The RT-PCR conditions were as instructed by the manufacturer's protocol. The resulting DNA fragments were analyzed by gel electrophoresis; the expected transgene product was $570 \mathrm{bp}$.

Western blot analysis. BAECs were cultured in 10-cm dishes and grown to subconfluence. Cells were infected with Ad-PPE-Fas-c or Ad-CMV-Fas-c, as described above. After lysis and evaluation of protein content (Micro BCA; Pierce Biotechnology Inc., Rockford, Illinois, USA), samples containing equal amounts of protein were separated by SDS-PAGE and transferred to Optitran BA-S83 reinforced nitrocellulose membranes (Schleicher \& Schuell BioScience Inc., Keene, New Hampshire, USA). The membranes were probed with rabbit anti-TNFR1 polyclonal antibody (Santa Cruz Biotechnology Inc., Santa Cruz, California, USA), followed by HRP-conjugated secondary antibodies and ECL reaction.

Cytotoxicity assays. The different cells were seeded in a 96-well microtiter plate at $10^{4}$ cells per $100 \mu \mathrm{l}$ per well. After an overnight incubation, cell monolayers were infected with the recombinant adenoviruses at the indicated MOI. Human TNF- $\alpha$ (SigmaAldrich, St. Louis, Missouri, USA) at the indicated concentrations was added to the culture media 48 hours after infection, and cell viability was assayed 72 hours after infection by crystal violet assay. Briefly, the surviving cells were stained with $1 \%$ crystal violet in water. The dye was eluted, and cell viability was reflected by dye absorbance, determined by absorbance measurements at $550 \mathrm{~nm}$, on an automated microplate reader.

Relative cell viability, in both assays, was calculated as follows: (experimental absorbance - background absorbance) $\times 100 /$ (absorbance of untreated control - background absorbance).

Animals. Twelve- to fourteen-week-old C57BL/6J mice $(n=14-16$ unless otherwise specified; Harlan Laboratories Ltd., Jerusalem, Israel) were used. All animal procedures were approved by the Animal Care and Use Committee of Sheba Medical Center.

Animal model of B16 melanoma. Melanoma cell monolayer in culture was washed and detached and then pelleted by brief centrifu- 


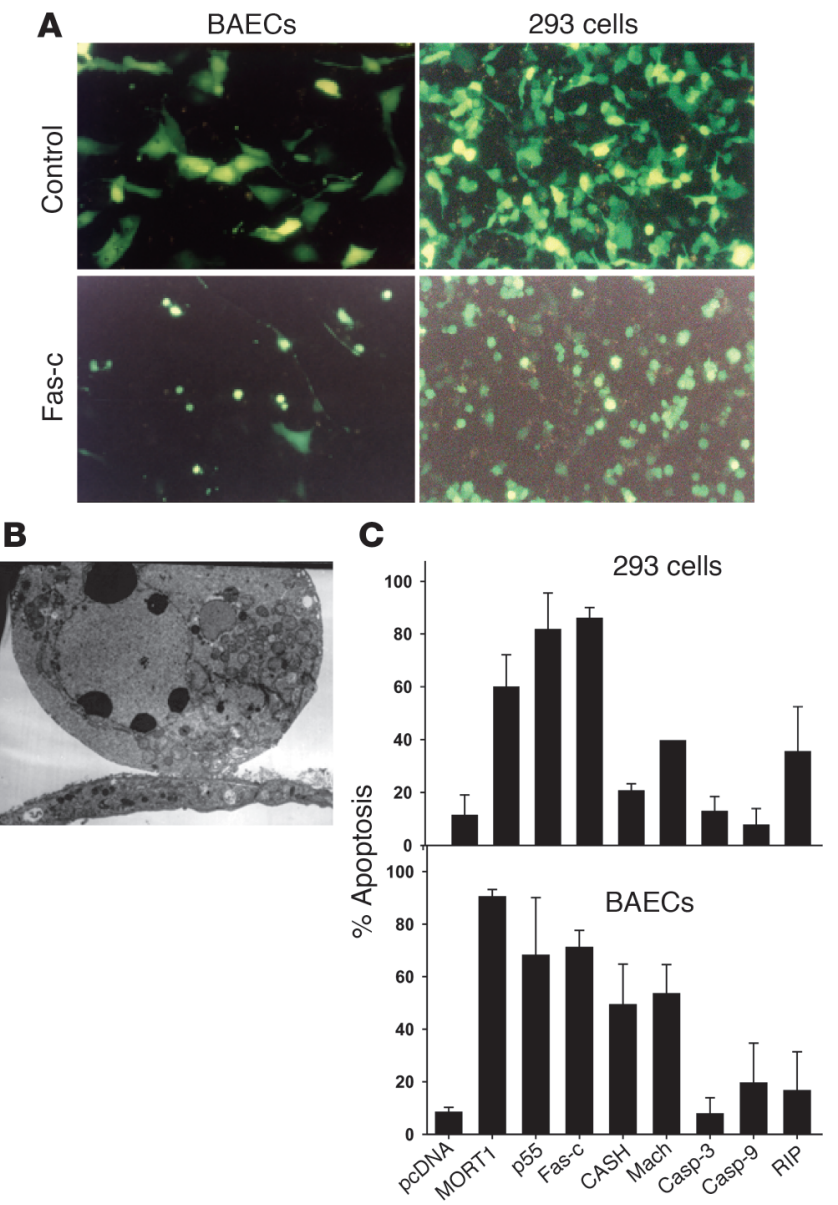

Figure 1

The activity of proapoptotic genes in ECs. (A) BAECs (left) or 293 cells (right) cotransfected with GFP plasmid and control plasmid (upper panels) or with GFP plasmid and Fas-c (lower panels). All magnifications are $\times 200$. (B) Electron microscopy of representative BAECs transfected with TNFR1, showing characteristic ultrastructural features of apoptosis, such as chromatin condensation into a few big round clumps. (C) Summary of the proapoptotic activity of MORT1 (FADD), TNFR1 (p55), Fas-c, CASH (c-FLIP), Mach (caspase-8), caspase-3 (casp-3), caspase-9 (casp-9), and RIP plasmids. The percentage of apoptotic cells from the total GFP-expressing cells was calculated. Each bar represents the mean $\pm S D$ of at least three experiments in triplicates.

gation at $100 \mathrm{~g}$. The supernatant was removed, cell pellets were resuspended in saline, and the cell number was counted. The cell concentration was finally adjusted to $7.5 \times 10^{6}$ cells $/ \mathrm{ml}$. Male C57BL/6J mice (at 3 months old) were anesthetized by isoflurane inhalation, and $100 \mu \mathrm{l}$ of the suspension was injected subcutaneously into left flank of mice. The tumor area was measured using a hand caliper and calculated by multiplication of length and width. When tumor area reached approximately $0.5 \mathrm{~cm}^{2}$, mice were randomly assigned to saline $(n=4)$ or Ad-PPE-Fas-c $(n=7)$ injection.

LLC model. D122-96 (LLC) cells were grown until ten $75-\mathrm{cm}^{2}$ flasks reached confluence. The cells were then trypsinized, and recovered from the medium by brief centrifugation. They were diluted in physiological saline to the concentration of $10^{7}$ cells $/ \mathrm{ml}$. Male C57BL/6J mice (at 3 months old) were anesthetized by isoflurane inhalation, and $5 \times 10^{5}$ cells (in a volume of $50 \mu \mathrm{l}$ ) were injected subcutaneously to their footpads. Primary tumors developed in the feet in about 14 days. When the tumor reached the diameter of $7 \mathrm{~mm}$, the mice were anesthetized with pentobarbital-Na (40 $\mathrm{mg} / \mathrm{kg}$ intraperitoneally), and the tumor was resected by amputation of the distal foot. Five and 14 days after primary tumor resection, either $10^{11}$ viral particles of one of the various viral vectors or $100 \mu \mathrm{l}$ of saline (control) was systemically injected into the tail veins. Following the excision of the primary tumors, metastases developed in the lungs of the mice. Once five of the control, salineinjected mice died of metastasis, every mouse that reached the same day after tumor resection on which the fifth control mouse died was sacrificed.

ELISA test. In order to assay the humoral response against the transgene following systemic administration, human soluble TNFR1 (PeproTech Inc., Rocky Hill, New Jersey, USA) was diluted in PBS, added to 96-well plates, and incubated overnight. After serial washings, wells were blocked with BSA and incubated with 1:25 dilution of mouse sera overnight. Goat anti-mouse IgG conjugated to alkaline phosphatase was diluted 1:5,000, applied, and left for 2 hours. p-nitrophenyl phosphate (PNPP) served for color development. Absorbance was read at $405 \mathrm{~nm}$.

Real-time PCR analysis of transgene biodistribution. Twenty-two male C57BL/6J mice (at 3 months old) were injected with D122-96 cells, and their primary tumor was resected, as before. Twenty-one days after amputation, mice were treated systemically (by tail-vein injection) with $2 \times 10^{11}$ of the viral particles in $200 \mu \mathrm{l}$ of saline. Six days after injection, mice were sacrificed and the following organs were frozen in liquid nitrogen: lung, liver, heart, stomach, small intestine, spleen, kidney, gonads, and brain. Viral DNA was extracted using the High Pure Viral Nucleic Acid Kit (Roche Diagnostics $\mathrm{GmbH}$ ). The PCR primers used for amplification of adenovirus 5 (Ad5) hexone were: upstream primer, $5^{\prime}$-TACGCCAACTCCGCCCACCGCGCT-3'; downstream primer, 5'-GCCGAGAAGGGCGTGCGCAGGTA-3'. $\beta$-Actin was used for normalization of results. The $\beta$-actin primers (for genomic DNA) were: upstream primer, $5^{\prime}$ CTCCTGAGCGCAAGTACTCC-3'; downstream primer, 5' -CTGCTTGCTGATCCACATCTG-3'. Fas-c mRNA was amplified by TaqMan real-time PCR using the upstream TNFR1 primer $5^{\prime}$ AGATCTCTTCTTGCACAGTG-3' and the downstream Fas primer 5'-TGAAGTTGATGCCAATTACG-3'. The results were normalized with $\beta$-actin mRNA and presented as copies per cell. Additionally, PCR products were loaded on a denaturing agarose gel. Real-time PCR analysis was done by LightCycler-FastStart DNA Master SYBR Green I (Roche Applied Science, Mannheim, Germany) according to the manufacturer's instructions.

TUNEL assay. TUNEL assay was performed on paraffin-embedded lung tissues using an ApopTag kit (Intergen Inc., Purchase, New York, USA).

Immunohistochemistry. Upon sacrificing, tumoral tissues were frozen in Tissue-Tek OCT compound (Sakura Finetek USA Inc., Torrance, California, USA) and cryosectioned. ECs were immunostained using rat monoclonal anti-CD31 antibodies (Pharmingen, San Diego, California, USA). The background was stained with hematoxylin.

Statistical methods. SigmaStat (SPSS Science, Chicago, Illinois, USA) was used for statistical analysis. One-way repeated-measures ANOVA was performed in the in vitro studies and for the LLC model, and Student's $t$ test was used for the B16 melanoma model; $P$ less than 0.05 was considered significant. Data are presented as mean \pm SEM and were graphed using SigmaStat or Microsoft Excel (Microsoft Corp., Redmond, Washington, USA). 


\section{Results}

Fas-c death receptor overexpression results in apoptosis in endothelial and nonendothelial cells. In order to identify the proapoptotic gene that is most potent in ECs, we screened several proapoptotic genes in vitro. Full cDNA of MORT1 (Fas-associated death domain protein [FADD]) (25), TNFR1 (p55), CASH (c-FLIP) (31), Mach (caspase-8), caspase-3, caspase-9, RIP, and Fas-c (containing the extracellular region of TNFR1 and the transmembrane and intracellular regions of Fas) $(25,32)$ was subcloned into the multiple cloning site of pcDNA3, under the control of the cytomegalovirus (CMV) promoter. The proapoptotic genes were cotransfected with pEGFP-C1 into BAECs. 293 cells were used as nonendothelial control. Twenty-four hours after transfection, the cells were analyzed using fluorescent microscopy. Apoptotic cells were identified by their typical morphology. When compared with normal cells, they appear smaller and rounder (Figure 1A). Cells transfected with the proapoptotic plasmids displayed typical appearance of apoptotic cells on electron microscopy (Figure 1B). MORT1, TNFR1, and Fas-c induced the highest apoptotic activity in both BAECs and 293 cells $(P<0.05$ compared with the control vector; Figure $1 \mathrm{C}$ ). Of these three, we chose to insert the Fas-c gene in an adenoviral vector to be used in vitro and in vivo. This gene triggers fast and efficient Fas-mediated apoptosis by binding TNF- $\alpha$, which is abundant in the tumor microenvironment (26) and circumvents the use of the toxic FasL (24).
Adenoviral vector containing Fas-c death receptor under the control of the $P P E-1-3 x$ promoter induces apoptosis specifically in ECs. We constructed a recombinant adenovirus containing the modified PPE- 1 promoter and a full-length Fas-c (Figure 2A). Transduction of ECs (BAECs and HUVECs) with this vector resulted in transgene mRNA transcription (Figure 2B) and dose-dependent Fas-c protein expression (Figure 2C). Infection of ECs, but not of nonendothelial cells, at an MOI of 1,000 induced massive cell death in the absence of the ligand TNF- $\alpha$ (Figure 2D). In contrast, the recombinant vector containing the Fas-c gene driven by the non-EC-specific CMV promoter (Ad-CMV-Fas-c) induced apoptosis in both endothelial and nonendothelial cells (Figure 2E). Infection of ECs at a lower MOI did not result in cell death. It did, however, sensitize the cells to the proapoptotic effect of TNF- $\alpha$ (Figure 2F). Nonendothelial cells, both primary and transformed, were not sensitized to TNF- $\alpha$ (data not shown). These in vitro results suggested that Ad-PPE-Fas-c, containing the Fas-c gene under the control of the modified PPE-1 promoter, is an efficient tool for EC execution. Its activity is independent of ligand at high levels of infection and dependent on TNF- $\alpha$ at lower levels of infection.

Biodistribution of transgene expression and antibody formation against the transgene. We next sought to establish that Fas-c controlled by the PPE-1-3x promoter reaches its metastasis-bearing target organ and is expressed there preferentially following systemic adminis-
A
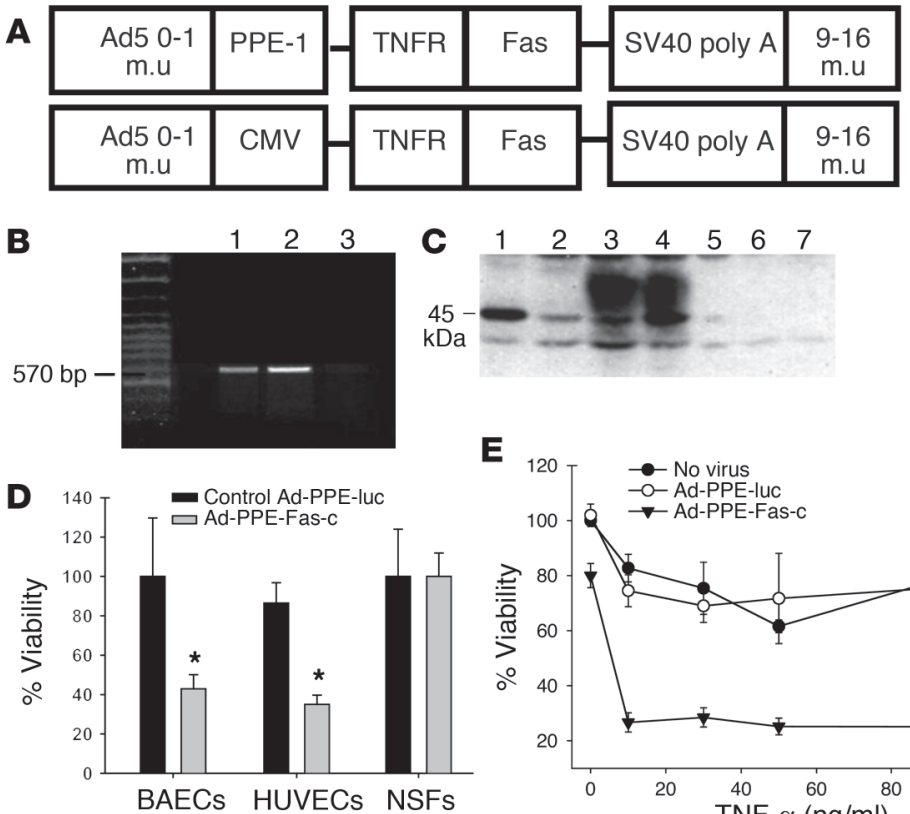

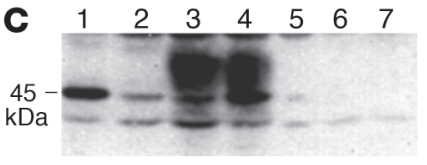

$\mathbf{E}$

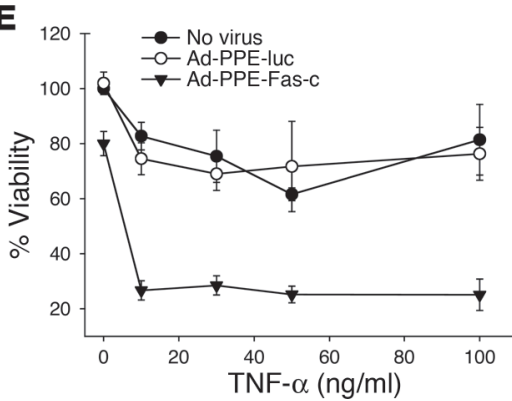

$\mathbf{F}$
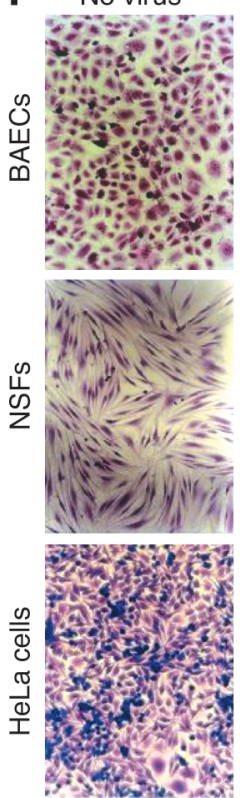

Figure 2

Construction and activity of PPE-directed Fas-c recombinant adenoviruses. (A) Schematic representation of the recombinant adenovirus early region 1-deleted (E1-deleted) vectors. m.u, map units; simian virus 40 (SV40 poly A). (B) RT-PCR analysis of BAECs infected by Ad-PPE-Fas-C. Lane 1: Fas-c plasmid, positive control. Lane 2: Ad-PPE-Fas-c virus. Lane 3: Ad-PPE-luc virus, negative control. (C) Western blot analysis of BAECs infected with Ad-PPE-Fas-c. Lanes 1 and 2: BAECs transfected with pcDNA3-Fas-c (positive control). Lanes 3 and 4: BAECs infected with Ad-PPE-Fas-c at MOI 100 and 1,000, respectively. Lane 5: Noninfected BAECs. Lanes 6 and 7: BAECs infected with Ad-PPE-luc at MOI 100 and 1,000 , respectively. (D) Crystal violet viability assay of endothelial cells (BAECs and HUVECs) and nonendothelial cells (NSFs) infected with Ad-PPE-Fas-c at MOI 1,000. Cells were infected with Ad-PPE-Fas-c or Ad-PPE-luc or were not infected. Each bar represents the mean \pm SD of four to six replicates of six to eight wells. ${ }^{*} P<0.05$ vs. control vector-infected cells. (E) Sensitization of ECs to TNF- $\alpha$ cytotoxicity by Ad-PPE-Fas-c infection. BAECs were infected with Ad-PPE-Fas-c or control virus. Human TNF- $\alpha$ at the indicated concentrations was added to the culture media. (F) The effect of Ad-CMV-Fas-c on apoptosis in ECs and nonendothelial cells. BAECs, NSFs, and HeLa cells were infected with Ad-PPE-Fas-c (center) or Ad-CMV-Fas-c (right) at MOI 1,000 and stained by crystal violet. Nonendothelial cells were unaffected by Ad-PPE-Fas-c; however, massive apoptosis was induced by Ad-CMV-Fas-c infection. $\times 200$. 

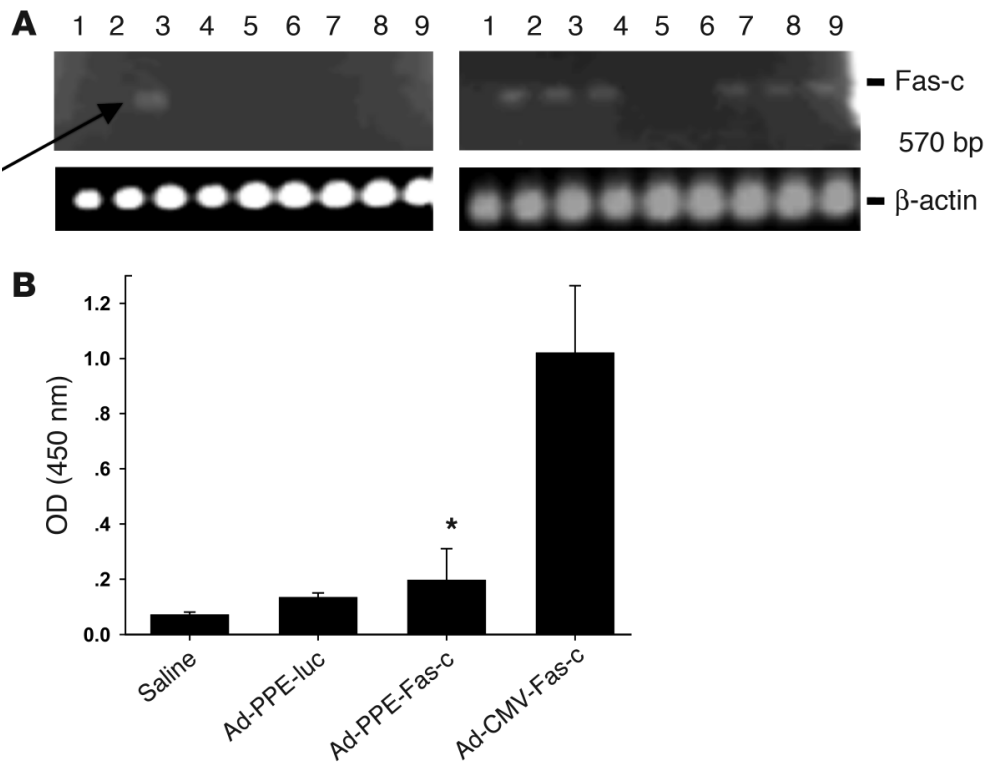

\section{Figure 3}

Specificity of Ad-PPE-Fas-c expression in LLC metastasis-bearing mice. (A) Biodistribution analysis. LLC lung metastases were created in male C57BL/6J mice $(n=22)$, and mice were injected with $2 \times 10^{11}$ of the viral vectors. Six days later, mice were sacrificed, and the various organs were snap-frozen in liquid nitrogen. RT-PCR analysis was done using upstream TNFR1 primer and downstream Fas primer. Lanes: 1, brain; 2, heart; 3, lung; 4, liver; 5, stomach; 6, small intestine; 7, spleen; 8, kidney; 9, gonads. Left: Fas-c expression in Ad-PPE-Fas-c-injected mouse. Arrow: restricted expression in the metastasis-bearing lungs. Right: Ad-CMV-Fas-c-injected mouse. (B) Transgene antibody titer. The various vectors were injected twice into LLC-bearing mice, at a 9-day interval. Plasmas were collected 10 days after the second injection, and ELISA was done for the detection of IgG antibodies against human TNFR1. Each bar represents the mean \pm SE, $n=8-10$. ${ }^{\star} P<0.05$ vs. Ad-CMV-Fas-c-injected mice. tration. Mice bearing LLC metastases were injected i.v. with $2 \times 10^{11}$ viral particles of Ad-PPE-Fas-c, Ad-CMV-Fas-c, Ad-PPE-luc, or saline. Six days later, their organs were subjected to RT-PCR analysis. Transcriptional control of Fas-c by PPE-1-3x led to expression restricted to the tumor-bearing lung, as demonstrated by gel electrophoresis (Figure 3A). In contrast, Ad-CMV-Fas-c-injected mice expressed Fas-c mRNA in most tissues tested. Real-time RT-PCR amplification of Ad5 hexone verified that, as expected, both PPE and CMV vectors reached all tissues tested, with the higher virus titer in the liver (data not shown).

One of the major limitations of adenovirus-mediated gene therapy is the transient transgene expression, as observed in various in
A

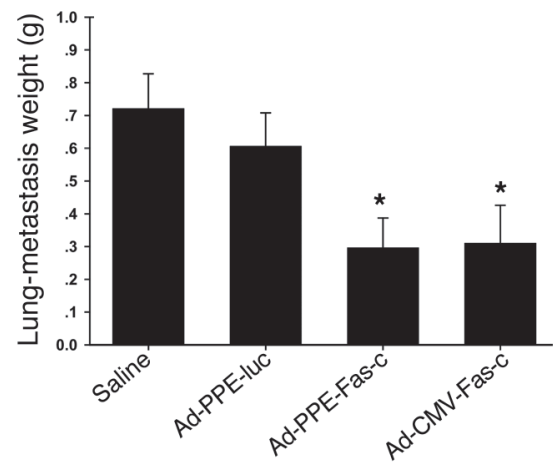

B Saline

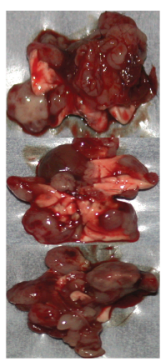

Ad-PPE-luc Ad-CMV-Fas-c Ad-PPE-Fas-c

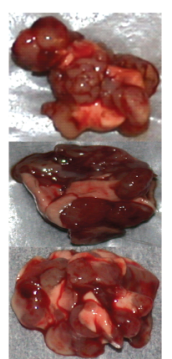

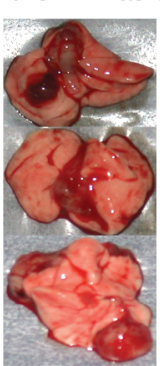

C

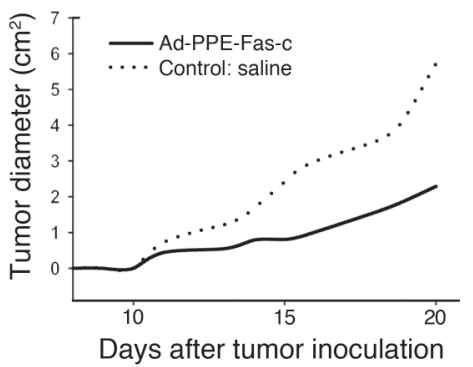

E

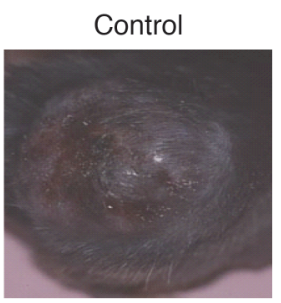

D

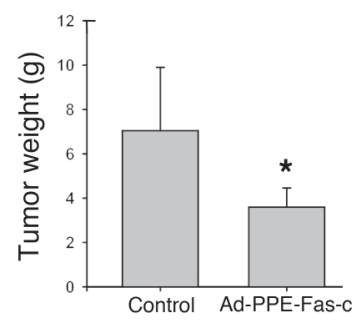

Ad-PPE-Fas-c

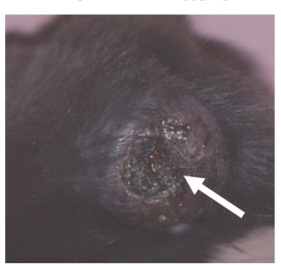

Figure 4

Systemic administration of Ad-PPE-Fas-c inhibited tumor growth. (A) LLC lung metastases were created in male mice. Ad-PPE-Fas-c, Ad-CMV-Fas-c, Ad-PPE-luc, or saline was injected i.v. into the tail vein. Weight due to tumor burden was calculated by subtraction of the average normal wet organ weight $(200 \mathrm{mg})$ from each tumor-bearing organ. Each bar represents the mean $\pm \mathrm{SE}, n=14-16$. ${ }^{*} P<0.05$ vs. control groups. (B) Representative lung surfaces of treated versus untreated mice. (C) Tumor growth kinetics. Male C57BL/6J mice were inoculated subcutaneously on the hind flank with $7.5 \times 10^{6}$ B16 melanoma cells. Saline $(n=4)$ or Ad-PPE-Fas-c $(n=7)$ was injected i.v. when tumor was palpable. Tumor diameter was calculated from tumor measurements scored at the indicated postimplantation day. The growth of B16 melanoma was significantly inhibited in mice treated with Ad-PPE-Fas-c compared with control mice. (D) B16 melanoma tumor weights at the day of sacrifice. Tumor weights were lower in mice injected with Ad-PPE-Fas-c. ${ }^{*} P<0.05$ vs. control group. (E) Prominent tumor necrosis in an Ad-PPE-Fas-c-treated mouse. Arrow: necrotic area. 

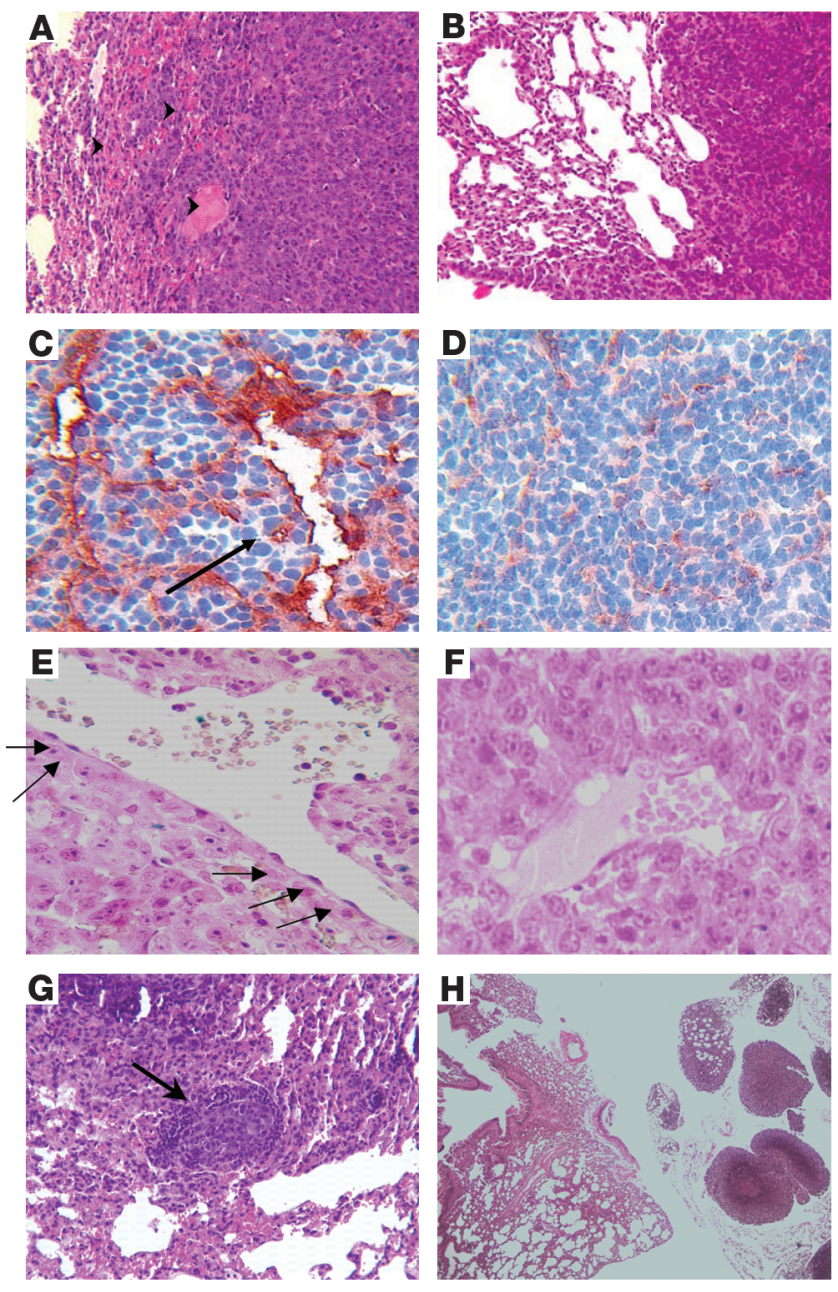

vivo models (15). Immunological responses to viral or transgene targets can eliminate transduced cells and cause the loss of transgene expression. It was demonstrated that immune responses directed against foreign transgene-encoded proteins are the major determinant of the duration of gene expression (16). The following experiment was intended to find out whether gene expression limited to ECs under the control of the PPE promoter results in less immunogenicity. Mice bearing LLC metastases were treated twice at a 9-day interval, via tail-vein injection, with the various vectors at the same concentrations, as described above. High antibody levels to TNFR1 were detected 10 days after injection in mice treated with Ad-CMV-Fas-c, while minimal levels were detected in mice injected with Ad-PPE-Fas-c (Figure 3B). Antibody titers against the adenovirus hexone were similar among the different virus-injected groups (data not shown).

Antitumoral activity of Ad-PPE-Fas-c. The antitumoral activity of Ad-PPE-Fas-c was tested in the model of B16 melanoma primary tumor and in the LLC metastasis model. Mice bearing lung metastases of LLC were i.v. injected twice, at a 9-day interval, with Ad-PPEFas-c or Ad-CMV-Fas-c. Control animals were injected with Ad-PPEluc or saline. Mice were sacrificed when five of the control, saline-treated mice died of metastases. Average lung-metastasis weights of mice injected with Ad-PPE-Fas-c or Ad-CMV-Fas-c were reduced by $56 \%$ compared with those of controls (Figure $4 \mathrm{~A}$ ). Lung surfaces of most treated mice were free of tumors or partly covered

\section{Figure 5}

Ad-PPE-Fas-c inhibits metastasis growth by antivascular action. Vascular impairment of tumor vessels in Ad-PPE-Fas-C-treated mice is shown. (A) H\&E staining of a lung section taken from an Ad-PPE-Fas-c-treated mouse, showing hemorrhagic areas at the border between normal lung and metastasis. Arrowheads: hemorrhagic area. $\times 100$. (B) H\&E staining of a control, saline-treated mouse. $\times 100$. (C) CD31 staining of tumor blood vessels in an Ad-PPE-Fas-C-treated mouse. Arrow: noncontinuous endothelial lining. $\times 200$. (D) CD31 staining of an Ad-CMV-Fas-C-treated mouse. $\times 200$. (E) TUNEL staining (marking apoptotic cells) of an Ad-PPEFas-c-treated mouse, showing apoptotic ECs in tumor blood vessels. Arrows: positive cells, stained with dark purple, lining a tumor blood vessel. $\times 400$. (F) TUNEL staining of a control, Ad-PPE-luc-treated mouse, demonstrating unstained vessels. $\times 400$. (G) Antitumor immune response. Micrometastases containing numerous apoptotic nuclei appear, surrounded by a mononuclear infiltrate, in this Ad-PPE-Fas-c-treated mouse. Arrow: mononuclear infiltrate. $\times 200$. (H) Small metastases restricted to the perihilar region in an Ad-PPE-Fas-c-treated mouse. $\times 40$.

by small, undeveloped metastases, while control animals' lungs were almost completely replaced by tumoral tissue (Figure 4B). To further test the therapeutic efficacy of this treatment, we randomly sorted mice bearing established, approximately $0.5-\mathrm{cm}^{2}$ B16 melanoma tumors into two groups and treated each group with a single tail-vein injection of saline, as a control, or Ad-PPE-Fas-c. Mice injected with Ad-PPE-Fas-c displayed significant retardation of tumor growth, starting at day 15 after tumor inoculation (Figure $4 \mathrm{C})$. Tumor weight on the day of sacrifice was significantly reduced compared with that in the control, saline-treated mice (Figure 4D). In addition, central tumor necrosis occurred more frequently and more massively in Ad-PPE-Fas-c-treated mice (Figure 4E).

The antiangiogenic mechanism of Ad-PPE-Fas-c antitumoral activity. To verify that Ad-PPE-Fas-c inhibited metastasis growth by antiangiogenic action, we performed histological analysis. Lung sections of Ad-PPE-Fas-c and of Ad-CMV-Fas-c were stained by H\&E as well as immunostained with anti-CD31 (anti-PECAM-1) for the detection of tumor blood vessels. In the PPE-controlled group, perivascular necrosis was noted inside metastasis, and hemorrhagic areas were dominant at the borders between lung and tumor tissue (Figure 5, A and B). Tumor blood vessel staining revealed giant thin-walled blood vessels in Ad-PPE-Fas-c-treated animals that were not detected in the CMV nor in the control groups. Moreover, the tumor blood vessels in the Ad-PPE-Fas-c group were damaged and had lost the endothelial layer (Figure 5, C and D). TUNEL staining, which marks apoptotic cells, demonstrated focal areas of apoptotic ECs and apoptotic cells in the nearby tumor in the Ad-PPE-Fas-c-treated group. In contrast, blood vessels of Ad-CMV-Fas-c treated mice and of control mice were unaffected. Positively stained tumor cells in these mice were detected almost exclusively in the metastasis periphery (Figure 5, E and F). In addition, micrometastases surrounded by a circular mononuclear infiltrate were detected in Ad-PPE-Fas-c-treated mice (Figure 5G) and, to a lesser extent, in Ad-CMV-Fas-c-treated mice (data not shown). Most of the small remaining metastases in the Ad-PPE-Fas-c group were confined to the perihilar region outside the lung (Figure 5H). Histological analysis of liver sections excluded antivascular action of Ad-PPE-Fas-c in normal, nonangiogenic tissues (Figure 6, A and B).

TNF- $\alpha$ administration results in liver necrosis in Ad-CMV-Fas-c-treated but not in Ad-PPE-Fas-c-treated mice. Finally, we sought to determine whether exogenous TNF- $\alpha$ administration to Ad-PPE-Fas-c-treated mice results in activation of the transgene protein in nontumoral 

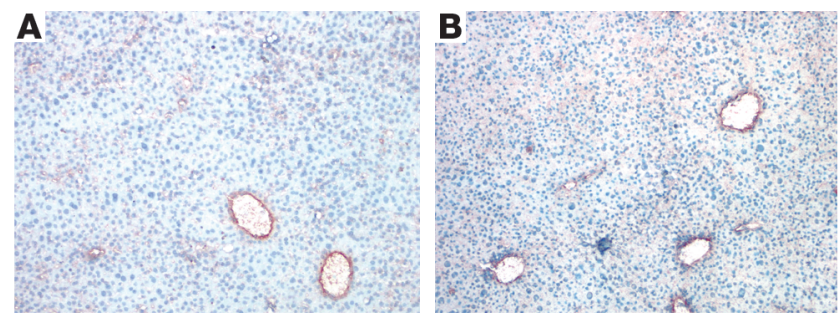

\section{Figure 6}

Angiogenesis specificity of Ad-PPE-Fas-c antivascular action. Liver sections from Ad-PPE-Fas-C-treated (A) or saline-treated mice $(\mathbf{B})$ were immunostained with anti-CD31 (anti-PECAM-1) antibodies. $\times 200$. Note the normal architecture of liver blood vessels.

tissues. Mice bearing LLC micrometastases were injected i.v. with the previously described vectors. Starting on the fourth day after viral injection, the mice received five i.v. doses of human TNF- $\alpha$, injected on 5 consecutive days. Ten days after the last TNF- $\alpha$ injection, the mice were sacrificed, and their liver histology was analyzed. Livers of the Ad-PPE-Fas-c group appeared almost normal, except for minimal trabecular unrest (Figure 7A). In contrast, Ad-CMVFas-c-treated mice exhibited massive liver necrosis (Figure 7B).

\section{Discussion}

Antiangiogenic therapy primarily targets the evolving vasculature, which nourishes the growing tumor. In the current work, by means of adenoviral gene transfer, we used a proapoptotic gene under the control of a modified EC- and angiogenesis-specific promoter, to selectively kill ECs of the emerging blood vessels feeding the tumor.

Targeting the endothelium of the tumor blood vessels is favored over targeting the tumor itself, as somatic mutations frequently occur in tumor cells, making the tumor resistant to apoptotic stimuli, including the Fas pathway (33). Additional advantages are enhanced viral spreading via the tumor's blood supply and an amplification effect on tumor killing, as one EC is known to support the nutritional needs of approximately 100 tumor cells (2).

In order to select the most potent proapoptotic gene for our adenoviral vehicle, genes from different stages of the apoptotic pathway were analyzed in ECs in vitro. We found that TNFR1, MORT1 (FADD), and Fas-c, the gene we ultimately chose to continue with, induced the highest apoptotic activity. The high degree of apoptotic activity displayed by Fas-c in both BAECs and HUVECs is in agreement with the findings of Kaplan et al., who demonstrated the susceptibility of human choriocapillaris ECs to FasL-presenting cells (21). For our purposes, this chimeric receptor (25) has an advantage in its ability to trigger the Fas apoptotic pathway following administration of TNF- $\alpha$, a less toxic substance than the native FasL. The toxicity of the native ligand was demonstrated by injection of recombinant FasL or anti-Fas mAb's into animals. Massive hepatocyte degeneration, necrosis, and hemorrhage were rapidly induced, ultimately leading to death $(24,34,35)$. Another advantage to the use of Fas-c in our adenoviral vector is the abundance of its ligand, TNF- $\alpha$, in the microenvironment of the tumor (26), which confers tumor specificity to the activity of the transgene. However, the activation of the Fas-c gene by endogenous TNF- $\alpha$ in vivo was not established in this study and should be examined in further experiments.

The advantage of using an endothelium-specific promoter over using a constitutive promoter for antitumoral gene therapy was confirmed in this research in two ways. First, we performed real- time PCR analysis of the viral genome and of the Fas-c transgene. While viral vectors were found in all tissues tested, we demonstrated that gene expression directed by the PPE-1-3x promoter is restricted to the target metastasis-bearing organ. In contrast, use of the CMV promoter resulted in nonspecific expression. Second, we showed that, by limiting gene expression to ECs, the PPE-1-3x promoter prevents the provocation of a humoral response against the transgene. As expected, the development of antibodies against the virus itself was not altered. However, the major determinant of gene-expression stability was found to be the immune response directed against the foreign transgene-encoded proteins, which was avoided in our case (16). This promoter might also be used with higher-generation "gutless" vectors that contain no viral coding sequences, thus abrogating the antiviral response. Several researchers have used EC-specific promoters, like E-selectin or vWF promoters, to deliver cytotoxic genes directly to the tumor vasculature $(6,36)$. However, these human promoters are weak and do not allow for high levels of expression. Their potential for use in vivo is therefore limited. In contrast, the PPE-1-3x promoter, although of a murine source, displays high levels of expression in both human and nonhuman ECs.

The specificity of the PPE promoter to angiogenic ECs over normal, nonactivated ECs was established by us previously using GFP- and luciferase-expressing vectors controlled by this promoter (12). In vitro, the PPE promoter was four times more activated in proliferating BAECs than in quiescent cells. In vivo experiments using the LLC model established that the PPE promoter led to about 200 times higher luciferase activity in lung metastasis than in nonvascularized primary tumor and naive lungs. In the metastatic lung, high levels of GFP expression were detected in small angiogenic vessels in the metastasis and no expression was detected in the normal lung tissue.

In the current work, we used a modified PPE promoter that contains three copies of the EC-positive regulatory elements, termed PPE-1-3x (13). Gene expression controlled by the PPE-1-3x promoter is even more efficient and more specific to activated ECs than is that controlled by the native, nonmodified PPE promoter (N. Varda-Bloom, manuscript submitted for publication).

Targeting of ECs can be done by using specific ligands or specific promoters. Recently, nanoparticles coupled to the $\alpha_{v} \beta_{3}$ integrin ligand and carrying a mutant Raf gene were shown to induce regression of tumors (37).
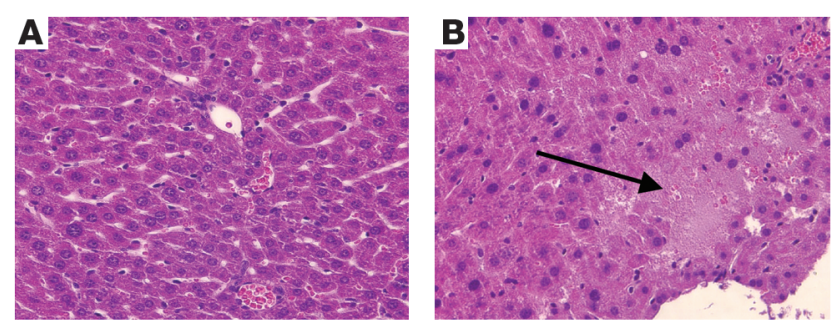

\section{Figure 7}

Liver necrosis induced by uncontrolled transcription of the Fas-c transgene. Liver sections of tumor-bearing mice demonstrate the nonspecific activity of Ad-CMV-Fas-c (B), compared with Ad-PPE-Fas-C (A). LLC lung metastases were created in 90 male mice, as before. AdPPE-Fas-c, Ad-CMV-Fas-c, Ad-PPE-luc, or saline was injected i.v. twice, 5 and 14 days after tumor resection, with human TNF- $\alpha$ (1 $\mu \mathrm{g} /$ mouse). Ten days later, mice were sacrificed and livers were stained. Arrow: broad necrotic area in Ad-CMV-Fas-c-injected mouse. $\times 200$. 
The antitumoral activity of the vector was assayed both on a model of primary tumor and on a lung-metastasis model. The LLC model was selected to prove our concept because of the rich vascularity of the developing metastases and because of the demonstrated response of this model to antiangiogenic therapy (38-40). Using this model, we demonstrated that PPE-1-3x-directed Fas-c elicits an impressive antitumoral effect. The mechanism of this effect, as suggested by the histological analysis, is direct damage to the tumor blood vessels, resulting in perivascular necrosis and hemorrhages. This antivascular effect appeared to be specific to the tumor, as the livers of treated animals were not affected even when exogenous TNF- $\alpha$ was administered to AdPPE-Fas-c-treated mice, whereas Ad-CMV-Fas-treated animals suffered severe liver necrosis.

In summary, we developed a system for transcriptional targeting of tumor vasculature. This system has several advantages over other approaches to antivascular gene therapy: (a) it makes use of a promoter that is expressed efficiently and selectively in angiogenic ECs, and (b) it uses a potent apoptosis-inducing gene activated by TNF- $\alpha$, a relatively tumor-specific ligand, providing a supplementary level of specificity to tumor angiogenesis. Efficient targeting of tumor blood vessels by gene therapy will most likely be achieved by a combination of transductional and transcriptional means, such as using endothelium-specific promoters.

\section{Acknowledgments}

This research was funded by the Israel Cancer Association through the Taecker fund Switzerland and by a grant from the Ministry of Health, The Division of Budgeting, Planning and Pricing. This work was done as part of thesis work by S. Greenberger in the Sackler Faculty of Medicine, Tel Aviv University. We thank Sylvie Polak-Sharcon for her help with the electron microscopy.

Received for publication November 25, 2003, and accepted in revised form January 28, 2004.

Address correspondence to: Dror Harats, Institute of Lipid and Atherosclerosis Research, Sheba Medical Center, Tel Hashomer 52621, Israel. Phone: 972-3-530-2940; Fax: 972-3-534-3521; E-mail: dharats@post.tau.ac.il.
1. Hanahan, D., and Folkman, J. 1996. Patterns and emerging mechanisms of the angiogenic switch during tumorigenesis. Cell. 86:353-364.

2. Denekamp, J. 1993. Review article: angiogenesis, neovascular proliferation and vascular pathophysiology as targets for cancer therapy. Br. J. Radiol. 66:181-196.

3. Claesson-Welsh, L., et al. 1998. Angiostatin induces endothelial cell apoptosis and activation of focal adhesion kinase independently of the integrin-binding motif RGD. Proc. Natl. Acad. Sci. U. S. A. 95:5579-5583.

4. Benjamin, L.E., and Keshet, E. 1997. Conditional switching of vascular endothelial growth factor (VEGF) expression in tumors: induction of endothelial cell shedding and regression of hemangioblastoma-like vessels by VEGF withdrawal. Proc. Natl. Acad. Sci.U.S. A. 94:8761-8766.

5. Jaggar, R.T., Chan, H.Y., Harris, A.L., and Bicknell, R. 1997. Endothelial cell-specific expression of tumor necrosis factor-alpha from the KDR or E-selectin promoters following retroviral delivery. Hum. Gene Ther. 8:2239-2247.

6. Ozaki, K., et al. 1996. Use of von Willebrand factor promoter to transduce suicidal gene to human endothelial cells, HUVEC. Hum. Gene Ther. 7:1483-1490.

7. Yanagisawa, M., Kurihara, H., Kimura, S., Goto, K. and Masaki, T. 1988. A novel peptide vasoconstrictor, endothelin, is produced by vascular endothelium and modulates smooth muscle Ca2+ channels. J. Hypertens. Suppl. 6:S188-S191.

8. Hu, J., Discher, D.J., Bishopric, N.H., and Webster, K.A. 1998. Hypoxia regulates expression of the endothelin1 gene through a proximal hypoxia-inducible factor- 1 binding site on the antisense strand. Biochem. Biophys. Res. Commun. 245:894-899.

9. Marsden, P.A., and Brenner, B.M. 1992. Transcriptional regulation of the endothelin-1 gene by TNFalpha. Am. J. Physiol. 262:C854-C861.

10. Kurihara, H., et al. 1989. Transforming growth factorbeta stimulates the expression of endothelin mRNA by vascular endothelial cells. Biochem. Biophys. Res. Commun. 159:1435-1440.

11. Harats, D., et al. 1995. Targeting gene expression to the vascular wall in transgenic mice using the murine preproendothelin-1 promoter. J. Clin. Invest. 95:1335-1344.

12. Varda-Bloom, N., et al. 2001. Tissue-specific gene therapy directed to tumor angiogenesis. Gene Ther. 8:819-827.

13. Bu, X., and Quertermous, T. 1997. Identification of an endothelial cell-specific regulatory region in the murine endothelin-1 gene. J. Biol. Chem. 272:32613-32622.

14. Armentano, D., et al. 1997. Effect of the E4 region on the persistence of transgene expression from adenovirus vectors. J. Virol. 71:2408-2416.

15. Verma, I.M., and Somia, N. 1997. Gene therapy: promises, problems and prospects. Nature. 389:239-242.

16. Tripathy, S.K., Black, H.B., Goldwasser, E., and Leiden, J.M. 1996. Immune responses to transgene-encoded proteins limit the stability of gene expression after injection of replication-defective adenovirus vectors. Nat. Med. 2:545-550.

17. Pastore, L., et al. 1999. Use of a liver-specific promoter reduces immune response to the transgene in adenoviral vectors. Hum. Gene Ther. 10:1773-1781.

18. Dengler, T.J., and Pober, J.S. 2000. Human vascular endothelial cells stimulate memory but not naive CD8+ T cells to differentiate into CTL retaining an early activation phenotype. J. Immunol. 164:5146-5155.

19. Dengler, T.J., Johnson, D.R., and Pober, J.S. 2001. Human vascular endothelial cells stimulate a lower frequency of alloreactive CD8+ pre-CTL and induce less clonal expansion than matching B lymphoblastoid cells: development of a novel limiting dilution analysis method based on CFSE labeling of lymphocytes. J. Immunol. 166:3846-3854.

20. Ashkenazi, A., and Dixit, V.M. 1998. Death receptors: signaling and modulation. Science. 281:1305-1308.

21. Kaplan, H.J., Leibole, M.A., Tezel, T., and Ferguson, T.A. 1999. Fas ligand (CD95 ligand) controls angiogenesis beneath the retina. Nat. Med. 5:292-297.

22. Volpert, O.V., et al. 2002. Inducer-stimulated Fas targets activated endothelium for destruction by antiangiogenic thrombospondin- 1 and pigment epithelium-derived factor. Nat. Med. 8:349-357.

23. Arai, H., Gordon, D., Nabel, E.G., and Nabel, G.J. 1997. Gene transfer of Fas ligand induces tumor regression in vivo. Proc. Natl. Acad. Sci. U. S. A. 94:13862-13867.

24. Schneider, P., et al. 1998. Conversion of membranebound Fas(CD95) ligand to its soluble form is associated with downregulation of its proapoptotic activity and loss of liver toxicity. J. Exp. Med. 187:1205-1213.

25. Boldin, M.P., et al. 1995. A novel protein that interacts with the death domain of Fas/APO1 contains a sequence motif related to the death domain. J. Biol. Chem. 270:7795-7798.

26. Khatib, A.M., et al. 1999. Rapid induction of cytokine and E-selectin expression in the liver in response to metastatic tumor cells. Cancer Res. 59:1356-1361.
27. Jaffe, E.A., Nachman, R.L., Becker, C.G., and Minick, C.R. 1973. Culture of human endothelial cells derived from umbilical veins. Identification by morphologic and immunologic criteria. J. Clin. Invest. 52:2745-2756.

28. Lifshitz, S., et al. 1998. Extensive apoptotic death of rat colonic cells deprived of crypt habitat. J. Cell. Physiol. 177:377-386.

29. McGrory, W.J., Bautista, D.S., and Graham, F.L. 1988. A simple technique for the rescue of early region I mutations into infectious human adenovirus type 5 . Virology. 163:614-617.

30. Bu, X., and Quertermous, T. 1997. Identification of an endothelial cell-specific regulatory region in the murine endothelin-1 gene. J. Biol. Chem. 272:32613-32622.

31. Goltsev, Y.V., et al. 1997. CASH, a novel caspase homologue with death effector domains. J. Biol. Chem. 272:19641-19644.

32. Hsu, H., Huang, J., Shu, H.B., Baichwal, V., and Goeddel, D.V. 1996. TNF-dependent recruitment of the protein kinase RIP to the TNF receptor-1 signaling complex. Immunity. 4:387-396.

33. Tamm, I., et al. 1998. IAP-family protein survivin inhibits caspase activity and apoptosis induced by Fas (CD95), Bax, caspases, and anticancer drugs. Cancer Res. 58:5315-5320.

34. Rensing-Ehl, A., et al. 1995. Local Fas/APO-1 (CD95) ligand-mediated tumor cell killing in vivo. Eur. J. Immunol. 25:2253-2258.

35. Ogasawara, J., et al. 1993. Lethal effect of the anti-Fas antibody in mice. Nature. 364:806-809.

36. Jaggar, R.T., Chan, H.Y., Harris, A.L., and Bicknell, R. 1997. Endothelial cell-specific expression of tumor necrosis factor-alpha from the KDR or E-selectin promoters following retroviral delivery. Hum. Gene Ther. 8:2239-2247.

37. Hood, J.D., et al. 2002. Tumor regression by targeted gene delivery to the neovasculature. Science. 296:2404-2407.

38. Kuo, C.J., et al. 2001. Comparative evaluation of the antitumor activity of antiangiogenic proteins delivered by gene transfer. Proc. Natl. Acad. Sci. U. S. A. 98:4605-4610.

39. Boehm, T., Folkman, J., Browder, T., and O'Reilly, M.S. 1997. Antiangiogenic therapy of experimental cancer does not induce acquired drug resistance. Nature. 390:404-407.

40. O'Reilly, M.S., Holmgren, L., Chen, C., and Folkman, J. 1996. Angiostatin induces and sustains dormancy of human primary tumors in mice. Nat. Med. 2:689-692. 